\title{
Assessment of the Effectiveness of Transcranial Magnetic Stimulation in Subjective Tinnitus
}

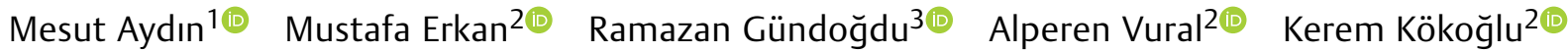 \\ Mehmet Illhan Şahin 20
}

\footnotetext{
1 Deparment of Otolaryngology, Kayseri City Hospital, Kayseri, Turkey

2 Deparment of Otolaryngology, Head and Neck Surgery, Faculty of Medicine, Erciyes University, Kayseri, Turkey

${ }^{3}$ Deparment of Otolaryngology, Derince Training and Research Hospital, Kocaeli, Turkey
}

Int Arch Otorhinolaryngol 2021;25(3):e453-e458.
Address for correspondence Kerem Kokoglu, Assistant Prof., Erciyes Üniversitesi Tıp Fakültesi Hastaneleri, KBB Hastalıkları Kliniği, Kayseri, 38039, Turkey (e-mail: dr.kokoglu@gmail.com).

\begin{abstract}
Introduction Increases in spontaneous activity in the neurons of the auditory cortex are presumed as the pathophysiology of tinnitus.

Objective To investigate the effectiveness of transcranial magnetic stimulation (TMS) in the treatment of tinnitus.

Methods A total of 62 patients between the ages of 22 and 74 with chronic subjective tinnitus were enrolled in the study. The inclusion criteria were normal findings on an otolaryngologic examination and normal ranges of complete blood count, routine biochemical parameter levels, thyroid function, air-bone gap on pure-tone audiometry, and middle-ear pressure and stapedial reflex on tympanometry. The patients were randomized into 2 groups: 40 patients were placed in the treatment for TMS (tTMS) group, and the rest (22 patients) were placed in the sham TMS group. The frequency and severity of the tinnitus, as well as the tinnitus handicap inventory (THI) score of each patient were measured before and one month after the treatment, and the values were statistically evaluated.

Results We observed a statistically significant improvement in the tTMS group $(p<0.05$ and $p<0.05$ respectively) regarding the THI and tinnitus severity scores. The sham TMS group showed no significant improvement in terms of the THI $(p>0.05)$. However, the tinnitus severity showed a significant increase in this group $(p<0.05)$. The comparison of differences in the changes in the THI and the tinnitus

Keywords

- sham group

- tinnitus

- tinnitus handicap inventory severity scores showed a significant statistical improvement in the tTMS group compared with the sham TMS group $(p<0.05$ and $p<0.05$ respectively).

Conclusion Transcranial magnetic stimulation was found to be effective in the treatment of tinnitus, and may be adopted as a treatment for tinnitus after further comprehensive studies.
\end{abstract}

received

February 12, 2020

accepted

August 13, 2020

published online

November 30, 2020
DOI https://doi.org/

10.1055/s-0040-1718530.

ISSN 1809-9777. (c) 2020. Fundação Otorrinolaringologia. All rights reserved.

This is an open access article published by Thieme under the terms of the Creative Commons Attribution-NonDerivative-NonCommercial-License, permitting copying and reproduction so long as the original work is given appropriate credit. Contents may not be used for commercial purposes, or adapted, remixed, transformed or built upon. (https://creativecommons.org/ licenses/by-nc-nd/4.0/)

Thieme Revinter Publicações Ltda., Rua do Matoso 170, Rio de Janeiro, RJ, CEP 20270-135, Brazil 


\section{Introduction}

Tinnitus is the perception of sound in the absence of auditory stimulation. ${ }^{1}$ The prevalence of tinnitus varies between $15 \%$ and $20 \%$ in the general population, and it causes a significant decrease in quality of life for $2 \%$ to $3 \%$ of the patients. ${ }^{2}$ In severe tinnitus, distress, ${ }^{3}$ depression, ${ }^{4}$ cognitive dysfunction $^{5}$ and insomnia are major debilitating factors that can interfere with the daily activities. ${ }^{6}$ The pathophysiological mechanism of tinnitus is still unknown. Many hypotheses have been put forward to explain the etiology of tinnitus. An aspect of these theories is that there is an electrical discharge in every nerve fiber, even in a resting condition, which causes spontaneous activity, and an increase in this spontaneous activity leads to tinnitus. ${ }^{7}$ The other hypothesis explained a connection between a peripheral auditory lesion inducing topsyturvy changes in the central neurological system due to active neuroplasticity. Although changes in the auditory web result in the perceptual characteristics of tinnitus, alterations in non-auditory regions are stressed to be liable for sensual and attentional transaction of the perception of tinnitus.

Several therapeutic approaches have been attempted to alleviate tinnitus, but the effectiveness of these treatments is inconsistent. Kim et $\mathrm{al}^{8}$ examined the efficacy of manual acupuncture, electroacupuncture and transcutaneous electrical nerve stimulation in the treatment of chronic tinnitus, and they found no improvement in the loudness and annoyance of tinnitus. Electroacupuncture reduced the tinnitus handicap inventory (THI) score significantly, in more than ten points. ${ }^{8}$

Low-frequency repetitive transcranial magnetic stimulation (TMS) of the auditory cortex has been proposed as a novel therapeutic tool for tinnitus. ${ }^{9}$ The TMS technique is noninvasive, and applies the principles of electromagnetism to carry an electrical current into the brain. Transcranial magnetic stimulation can suppress tinnitus by modulating the excitability of the cerebral cortex both in directly-stimulated and functionally-connected regions. ${ }^{10-12}$ Many clinical studies have shown that repeated (1,200 to 2,000 pulses/day for 5 to 10 days) low-frequency TMS at the temporoparietal lobe can reduce tinnitus. ${ }^{13}$ In the present study, we aimed to evaluate the therapeutic effects of TMS on tinnitus.

\section{Methods}

\section{Patients}

The patients who visited the otolaryngology department at a tertiary hospital with tinnitus complaints between February 2012 and July 2012 were recruited for the present study. All of the patients were enrolled after providing written informed consent. The study was approved by the local ethics committee, and was performed according to Helsinki Declaration (Decision Number: 2011/386). Patients who had subjective tinnitus for at least six months were included in study.

Patients with the following conditions were excluded from the study: ear infections, anatomic ear problems, Meniere disease, epilepsy, and severe psychiatric or chronic diseases. Additionally, patients with cardiac pacemakers or other implanted tools, and patients who were pregnant or breastfeeding were excluded.

All of the patients underwent detailed audiological, otolaryngological and neurological examinations to rule out treatable causes of tinnitus. The audiological examination included pure-tone audiometry and high-frequency audiometry. The matching of the tinnitus was performed by presenting sounds to the ear in which the tinnitus was not heard to determine the loudness and frequency of the tinnitus. The sample was ramdomly (by coin flip) divided into two groups: the TMS treatment group (tTMS) and the sham TMS group.

\section{TMS Conduction}

A Neuro-MS (Neurosoft, Ivanovo, Russia) TMS machine was used to conduct the TMS, which was performed using an 8shape (butterfly) probe on the left temporoparietal lobe at a low frequency $(1 \mathrm{~Hz}$ ) for 30 minutes; 40 patients underwent this treatment. The intensity of the stimulation was set at $100 \%$ of the motor threshold, consisting of 1,500 pulses. The 8 -shape probe only touched the left side of head of 22 patients at a $45^{\circ}$ angle from the midsagittal line, according to the description made by Ridding and Rothwell. ${ }^{14}$ There was no stimulation. Each patient completed five sessions in five days. During the TMS sessions, the ears of the patients were protected from the TMS noise using earplugs. The patients were not informed about the group in which they would be placed.

\section{THI (Tinnitus Handicap Inventory)}

The THI, which consists of 25 questions with 3 possible answers ("yes", "no" or "sometimes") was used. To each of these answers, 4, 0 and 2 points are assigned respectively. The overall score on the inventory ranges from 0 to 100 . With the THI, the degree of the complaints and their effects on the patients could be statistically evaluated, and the frequency of the tinnitus and the THI were determined just before first TMS session, and one month after the last session. The THI assessment and the TMS were performed by different researchers. Thus, the present study had a prospective, randomized, double-blinded, controlled design.

\section{Statistical Analysis}

The calculations were performed using the Statistical Package for the Social Sciences (SPSS, SPSS, Inc., Chicago, IL, US) software package, version 15.0. Compliance with the normal distribution of the data was evaluated using the ShapiroWilk test. Assessments of the loudness levels and frequencies of the tinnitus as well as the THI scores were performed using $t$-tests, paired $t$-tests, Mann-Whitney U tests and Wilcoxon tests. Values of $p<0.05$ were used to determine the statistical significance.

\section{Results}

In total, 62 tinnitus patients who had their data analyzed were recruited for the present study. Out of those patients, 
Table 1 Distribution of the study groups in terms of age, gender, symptoms and PTA results

\begin{tabular}{|l|l|l|l|l|}
\hline & tTMS Group & Sham Group & $p$-value & \multirow{2}{*}{0.966} \\
\hline Age (years) & & $49.47 \pm 14.16$ & $49.31 \pm 12.72$ & 0.271 \\
\hline \multirow{3}{*}{ Gender } & Female & $16(40 \%)$ & $12(54.5 \%)$ & \\
\cline { 2 - 4 } & Male & $24(60 \%)$ & $10(45.5 \%)$ & \\
\hline \multirow{2}{*}{ Symptom side } & Left Ear & $18(45 \%)$ & $8(36.3 \%)$ & 0.735 \\
\cline { 2 - 4 } & Right Ear & $11(27.5 \%)$ & $6(27.3 \%)$ & \\
\cline { 2 - 4 } & Bilateral & $11(27.5 \%)$ & $8(36.3 \%)$ & \\
\hline \multirow{2}{*}{ Meamptom duration (months): median (25-75\%) } & & $24(12-38)$ & $27(12-48)$ & 0.690 \\
\hline
\end{tabular}

Abbreviations: PTA, pure-tone audiometry; tTMS, treatment for transcranial magnetic stimulation.

40 were placed in the tTMS group, and 22 were placed in the sham TMS group; 28 (45.2\%) of the patients were women, and 34 (54.8\%) were men, and their mean age was $49.41 \pm 13.56$ years (range: 22 to 74 years). In total, 19 (30.6\%) patients had bilateral tinnitus, 17 (27.4\%) were affected in the right ear, and 26 (41.9\%) were affected in the left ear. The groups were similar in terms of age, gender, mean symptom duration, symptomatic ear side and puretone audiometry results.

The differences in THI scores before and after the TMS treatment were evaluated within each group. We observed that the change in the THI score in the tTMS group (before TMS: median - 46 [range: 32 to 68]; after TMS: median - 41 [range: 14.5 to 59 ]) was statistically significant $(p<0.05)$, while the change in the THI score in the sham TMS group (before TMS: median - 56 [range: 7.5 to 69]; after TMS: median - 57 [range: 17.5 to 70.5]) was statistically insignificant $(p=0.273)$. When the groups were compared, no sta- tistically significant differences were detected regarding the THI scores before and after the treatment (before TMS, $p=0.658$; after TMS, $p=0.161$ ) (-Table 1). When the THI score changes were compared between the groups (median scores: tTMS group - 4 [range: 0.5 to 16 ]; sham TMS group 0 [range: -2 to 0 ]), there was a significant difference $(p<0.05)$ (-Table 2).

The change in tinnitus loudness before and after the TMS treatment was evaluated within each group. We found that the difference in loudness in the tTMS group (before TMS: median - $65 \mathrm{~dB}$ [range: 46.25 to 75.75]; after TMS: median $65 \mathrm{~dB}$ [range: 45 to 74.25 ]) was statistically significant $(p<0.05)$, and the difference in loudness in the sham group (before TMS: median - 52.5 [range: 33.75 to 70]; after TMS: median - 55 [range: 36.5 to 70 ]) was statistically significant, with an unfortunate increase in loudness $(p<0.05)$ (-Table 2). When the differences regarding the change in loudness were compared between the groups (tTMS group:

Table 2 Comparison of the study groups in terms of differences in tinnitus frequencies and THI scores (before and after TMS)

\begin{tabular}{|c|c|c|c|c|}
\hline & $\begin{array}{l}\text { tTMS Group: median } \\
(25-75 \%)\end{array}$ & $\begin{array}{l}\text { Sham Group: median } \\
(25-75 \%)\end{array}$ & $p$-value & \\
\hline \multirow[t]{3}{*}{ Tinnitus loudness (dB) } & Before TMS & $65(46.25-75.75)$ & $52.5(33.75-70)$ & 0.037 \\
\hline & After TMS & $65(45.25-74.75)$ & $55(36.50-70)$ & 0.429 \\
\hline & $p$-value* & $<0.05$ & & \multirow[b]{2}{*}{$<0.05$} \\
\hline $\begin{array}{l}\text { Changes in differences in } \\
\text { tinnitus loudness }\end{array}$ & & $0(0-10)$ & $0(-5-0)$ & \\
\hline \multirow[t]{3}{*}{ Tinnitus frequency (kHz) } & Before TMS & $6(4-6)$ & $6(2.4-6)$ & 0.841 \\
\hline & After TMS & $6(4-6)$ & $6(4-8)$ & 0.334 \\
\hline & $p$-value ${ }^{* *}$ & 0.189 & 0.174 & \\
\hline $\begin{array}{l}\text { Change in difference in } \\
\text { tinnitus frequency }\end{array}$ & & $0(-2-0)$ & \multicolumn{2}{|l|}{0.823} \\
\hline \multirow[t]{3}{*}{ THI Score } & Before TMS & $46(32-68)$ & $56(17.5-69)$ & 0.658 \\
\hline & After TMS & $41(14.5-59)$ & $57(17.5-70.5)$ & 0.160 \\
\hline & $p$-value ${ }^{* * *}$ & $<0.05$ & 0.273 & \\
\hline THI score: change in difference & & $4(0.5-16)$ & $0(-2-0)$ & $<0.05$ \\
\hline
\end{tabular}

Abbreviations: THI, tinnitus handicap inventory; TMS, transcranial magnetic stimulation; tTMS, treatment for transcranial magnetic stimulation. 
median - 0 [range: 0 to 10 ]; sham TMS group -0 [range: -5 to $0])$, there was a significant difference $(p<0.05)$ ( - Table 2 ).

When the tinnitus frequencies were compared before and after the TMS treatment, we found that the differences in frequencies in both groups were statistically insignificant (tTMS group: $p=0.189$; sham TMS group: $p=0.074$ ). When both groups were compared, no statistically significant differences were detected in the frequency values before $(p=0.841)$ and after the treatment $(p=0.334)(-$ Table 2$)$. Regarding the comparison of the differences in the change in tinnitus frequencies (tTMS group: median -0 [range: $-2,000$ to 0]; sham TMS: median - 0 [range: $-2,000$ to 0 ]), there was no significant difference $(p=0.823$ ) (-Table 2 ).

All of the patients were reassessed one month after the TMS treatment. In the tTMS group, 17 (42.5\%) out of 40 patients reported full recovery, and $3(7.5 \%)$ patients reported partial recovery. In the sham group (22 patients), only $2(9 \%)$ of the patients reported full recovery. Full recovery meant that they reported they no longer had symptoms of tinnitus.

In total $3(7,5 \%)$ patients in tTMS group reported temporary localized headaches in the temporal region that did not cause too much discomfort. The headaches disappeared at the end of the treatment, and no other side effects were reported.

\section{Discussion}

In the present study, we applied $1-\mathrm{Hz}$ TMS (1,500 stimulations) to 40 patients over the course of 5 days, with a 30 minute session held each day. We used a placebo treatment (sham tTMS) on 22 patients. This sham procedure was conducted by the researchers, who placed the probes on the heads of the patients at a $45^{\circ}$ angle. ${ }^{15}$ We assessed the patients before the treatment and one month after using the THI and tinnitus severity results. We accepted the THI as the gold standard to assess the patients, and it indicated a statistically significant recovery in the patients submitted to TMS ( $p<0.05)$, and no recovery in the patients in the sham TMS group $(p=0.273)$. A statistically significant decrease was observed in the severity of the tinnitus in the tTMS group $(p<0.05)$. When the THI and the differences in the change in tinnitus severity were compared, a statistically significant recovery was found $(p<0.05)$ : according to our results, the TMS treatment is statistically more successful than the sham treatment.

Several techniques are used to measure tinnitus. The frequency, severity and masking of the tinnitus are the most common methods. The reliability of the tinnitus frequency changes from patient to patient. The frequency can present differences from day to day or on the same day; although the tinnitus frequency is said to be stable, it is quite difficult to correctly perform frequency matching. ${ }^{7}$ Sönmez et al. ${ }^{16}$ stated that tinnitus frequencies for all patients ranged from $250 \mathrm{~Hz}$ to $10,000 \mathrm{~Hz}$, with a mean of $5,790.44 \pm 2,399.31 \mathrm{~Hz}$. Yllmaz et al. ${ }^{17}$ reported an average tinnitus frequency of $5796.67 \mathrm{~Hz}$. In the present study, we did not observe significant changes in average tinnitus frequencies posttreatment in both groups. In addition, we did not find associations between the patients' characteristics and their tinnitus frequencies.

The correct determination of tinnitus severity is a reliable method to assess the efficacy of the treatment. ${ }^{18}$ In the literature, the threshold for tinnitus severity has generally been reported to be over $0 \mathrm{~dB}$ to $15 \mathrm{~dB} .{ }^{19}$ In the present study, the lowest tinnitus severity found was $15 \mathrm{~dB}$, while the highest was $95 \mathrm{~dB}$; the severity was between $0 \mathrm{~dB}$ and $10 \mathrm{~dB}$ greater than the hearing thresholds in 59 patients, and between $11 \mathrm{~dB}$ and $15 \mathrm{~dB}$ greater in 3 patients. In the present study, while the severity of the tinnitus in the tTMS group decreased significantly, a significant increase was observed in the sham TMS group. However, some of these increases and decreases did not completely match the clinical recovery of the patients. While some patients felt more comfortable when their severity increased, other patients did not feel more comfortable, despite their decrease in severity. Thus, we think that tinnitus severity might not be a very suitable method to assess the comfort of the patients.

Figueireido et al. ${ }^{20}$ reported acoustic tests to be insufficient for the diagnosis of and follow-up care for tinnitus. ${ }^{20}$ Thus, scales that assess how the tinnitus is perceived psychosomatically have gained importance. It is possible to obtain information about the severity of the tinnitus by using various scales. However, most of these scales are not supported by reliability and validity studies. The most reliable and commonly used scale is the THI, which has been reported to be a highly consistent and reliable inventory for the assessment of the symptoms experienced by tinnitus patients. ${ }^{21}$ In the present study, we used the THI and accepted it as the criterion for recovery. While a statistically significant decrease was observed in the tTMS group, no difference was found in the sham TMS group. The answers to the question "Have you recovered?", given by the patients as "yes" or "I've felt quite a lot of comfort", were in parallel with the THI results. In all of the 17 patients in the tTMS group who reported recovery or relaxation, an obvious decrease in the THI score was observed.

Several tinnitus treatment methods have been attempted. The general success rates of the methods in the literature range from $30 \%$ to $80 \%$, and the effectiveness of placebo treatments has been mentioned in most of these studies. ${ }^{2}$

Recently, TMS has been reported as a promising method in the treatment of tinnitus. ${ }^{22}$ Functional imaging methods have shown an increase in activation in the left lower frontal and left temporal regions in patients with tinnitus. ${ }^{23}$ In positron emission tomography (PET) studies that did not consider the direction of the tinnitus, cortical activation was observed in the left hemisphere. ${ }^{24}$ Marcondes et al. ${ }^{25}$ separated 20 tinnitus patients into 2 groups, and submitted 1 group for 5 days to $1-\mathrm{Hz}$ TMS (1,020 stimulations/day), while the other group underwent sham TMS. A recovery rate of $50 \%$ was observed in the tTMS group, and no recovery was observed in the sham TMS group. In 2005, De Ridder et al. ${ }^{2}$ submitted 114 patients with unilateral tinnitus to 1 session of TMS, and reported that $25 \%$ of them experienced full recovery, and $28 \%$ experienced partial recovery. In their study in 2007, Kleinjung et al. ${ }^{26}$ submitted the patients to $1-\mathrm{Hz}$ TMS (2,000 stimulations/day) sessions for 10 days, and 
reported a $40 \%$ success rate 3 months later. In a study conducted with 32 patients in 2008, Langguth et al. ${ }^{27}$ reported that there were no differences between low-frequency and high-frequency TMS sessions, and both yielded positive results. In their study with 56 patients, Khedr et al. ${ }^{28}$ submitted the patients $1-\mathrm{Hz}, 10$ $\mathrm{Hz}$, and $25-\mathrm{Hz}$ (1,500 stimulations/day) TMS sessions for 10 days, and observed a significant recovery in all 3 groups 4 months later. Minami et al. ${ }^{22}$ submitted 16 patients to $1-\mathrm{Hz}$ TMS (1,200 stimulations/day) sessions; they reported positive results, and suggested that further studies on this treatment should be conducted. In 2010, Lorenz et al. ${ }^{29}$ submitted 10 patients to $1-\mathrm{Hz}$ tTMS sessions for 5 days, and obtained positive results. They reported that TMS might be more efficient if applied for a longer period of time. In 2003, Plewnia et al. ${ }^{30}$ applied $10-\mathrm{Hz}$ TMS to different parts of the head, and only reported positive results for 8 out of 14 patients. In another study in 2007, Plewnia et al. ${ }^{31}$ found PET activations in the brains of 6 patients (left temporoparietal in 3 patients, right temporoparietal cortex in 3 patients), and applied 1-Hz TMS (1,800 stimulations/session) to those areas for 10 days, and observed a significant recovery in their patients. Rossi et al. ${ }^{32}$ applied 1-Hz TMS (1,200 stimulations/day) to the left secondary hearing cortexes of 16 patients for 5 days, and observed a significant decrease in the tinnitus. Lee et al. ${ }^{33}$ applied $0.5-\mathrm{Hz}$ TMS (600 stimulations/session) for 5 days, but did not observe a significant decrease. Kleinjung et al. ${ }^{24}$ submitted 14 patients with tinnitus to PET, and observed maximal activation in the left temporal cortexes of 12 patients and in the right temporal cortexes of 2 patients. They applied $1-\mathrm{Hz}$ TMS (2,000 stimulations/session) to the area over the course of 5 sessions, and found that a significant decrease in the tinnitus remained 6 months later. While Kleinjung et al. ${ }^{26}$ reported that the duration of the tinnitus and hearing loss had a negative effect on the outcome of the TMS treatment, Minami et al. ${ }^{22}$ reported that these did not have an effect on the treatment outcome, and that tinnitus developing as a result of sudden hearing loss was resistant to TMS treatment. We did not observe any obvious effects of the duration of the tinnitus or hearing loss on clinical recovery. A total of $50 \%$ of the patients who benefited from the treatment had normal hearing, while only 1 of our patients with sudden hearing loss responded to the treatment.

Wang et al. ${ }^{34}$ observed a significant reduction in the loudness of the tinnitus after active low-frequency TMS treatment. They also found that this suppression was especially shown in subjects with normal hearing and without sleeping problems.

In a Cochrane Database study, Meng et al. ${ }^{35}$ reported that TMS is a safe treatment for tinnitus in the short-term. Yet, there were no adequate data to provide any grounds for the reliance of this treatment in the long-term.

Rossi et al. ${ }^{32}$ do not mentioned the side effects of lowfrequency TMS. They observed temporary localized headaches in the temporal region that did not cause too much discomfort as in 3 of our 40 patients. The headaches disappeared at the end of the treatment, and no other side effects were reported.

The comparatively higher recovery rate could be related to the shorter follow-up time ( 1 month), and to the fact that
TMS is a mystic treatment. The shorter follow-up time was one of the weaknesses of the present study. A longer followup could be better to evaluate the recovery of the patients. Another weakness was that we think it would have been better to have divided the groups into subgroups, such as patients with hearing loss, with sleep disturbance etc.

\section{Conclusions}

We believe that new treatments for tinnitus should be researched, since there is still no effective treatment method, and because some patients face serious problems because of it. We concluded that tTMS, which is a new approach, could be a very promising method, and with wider and more comprehensive studies, it could play a very important role in the treatment of tinnitus.

\section{Conflict of Interests}

The authors have no conflict of interests to declare.

\section{References}

1 Jastreboff PJ, Hazell JW. A neurophysiological approach to tinnitus: clinical implications. Br J Audiol 1993;27(01):7-17

2 De Ridder D, Verstraeten E, Van der Kelen K, et al. Transcranial magnetic stimulation for tinnitus: influence of tinnitus duration on stimulation parameter choice and maximal tinnitus suppression. Otol Neurotol 2005;26(04):616-619

3 Andersson G. Tinnitus loudness matchings in relation to annoyance and grading of severity. Auris Nasus Larynx 2003;30(02): 129-133

4 Langguth B, Landgrebe M, Kleinjung T, Sand GP, Hajak G. Tinnitus and depression. World J Biol Psychiatry 2011;12(07):489-500

5 Hallam RS, McKenna L, Shurlock L. Tinnitus impairs cognitive efficiency. Int J Audiol 2004;43(04):218-226

6 Crönlein T, Langguth B, Geisler P, Hajak G. Tinnitus and insomnia. Prog Brain Res 2007;166:227-233

7 Jastreboff P, Gray W, Mattox D. Tinnitus and hyperacusis. Otolaryngol Head Neck Surg 1998;165:3198-3222

$8 \mathrm{Kim}$ BH, Moon YK, Kim MH, Nam HJ. Comparing the effects of manual acupuncture, electroacupuncture, and transcutaneous electrical nerve stimulation on chronic tinnitus: a randomized controlled trial. Integr Med Res 2020;9(02):100409

9 Kleinjung T, Vielsmeier V, Landgrebe M, Hajak G, Langguth B. Transcranial magnetic stimulation: a new diagnostic and therapeutic tool for tinnitus patients. Int Tinnitus J 2008;14(02):112-118

10 Folmer RL, Carroll JR, Rahim A, Shi Y, Hal Martin W. Effects of repetitive transcranial magnetic stimulation (rTMS) on chronic tinnitus. Acta Otolaryngol Suppl 2006;126(556):96-101

11 Oberman L, Edwards D, Eldaief M, Pascual-Leone A. Safety of theta burst transcranial magnetic stimulation: a systematic review of the literature. J Clin Neurophysiol 2011;28(01):67-74

12 Schecklmann M, Lehner A, Gollmitzer J, Schmidt E, Schlee W, Langguth B. Repetitive transcranial magnetic stimulation induces oscillatory power changes in chronic tinnitus. Front Cell Neurosci 2015;9:421

13 Eichhammer P, Hajak G, Kleinjung T, Landgrebe M, Langguth B. Functional imaging of chronic tinnitus: the use of positron emission tomography. Prog Brain Res 2007;166:83-88

14 Ridding MC, Rothwell JC. Is there a future for therapeutic use of transcranial magnetic stimulation? Nat Rev Neurosci 2007;8(07): 559-567

15 Langguth B, Kleinjung T, Marienhagen J, et al. Transcranial magnetic stimulation for the treatment of tinnitus: effects on cortical excitability. BMC Neurosci 2007;8(01):45 
16 Sönmez O, Külahlı I, Vural A, Şahin Mİ, Aydın M. The evaluation of ozone and betahistine in the treatment of tinnitus. Eur Arch Otorhinolaryngol 2013;270(07):1999-2006

17 Yilmaz I, Akkuzu B, Çakmak O, Ozlüoglu LN. Misoprostol in the treatment of tinnitus: a double-blind study. Otolaryngol Head Neck Surg 2004;130(05):604-610

18 Leske MC. Prevalence estimates of communicative disorders in the U.S. Language, hearing and vestibular disorders. ASHA 1981; 23(03):229-237

19 Goodwin PE, Johnson RM. The loudness of tinnitus. Acta Otolaryngol 1980;90(5-6):353-359

20 Figueiredo RR, Azevedo AA, Oliveira PdeM. Correlation analysis of the visual-analogue scale and the Tinnitus Handicap Inventory in tinnitus patients. Rev Bras Otorrinolaringol (Engl Ed) 2009;75 (01):76-79

21 Aksoy S, Firat Y, Alpar R. The Tinnitus Handicap Inventory: a study of validity and reliability. Int Tinnitus J 2007;13(02):94-98

22 Minami SB, Shinden S, Okamoto Y, et al. Repetitive transcranial magnetic stimulation (rTMS) for treatment of chronic tinnitus. Auris Nasus Larynx 2011;38(03):301-306

23 Dierks T, Linden DE, Jandl M, et al. Activation of Heschl's gyrus during auditory hallucinations. Neuron 1999;22(03):615-621

24 Kleinjung T, Eichhammer P, Langguth B, et al. Long-term effects of repetitive transcranial magnetic stimulation (rTMS) in patients with chronic tinnitus. Otolaryngol Head Neck Surg 2005;132(04): 566-569

25 Marcondes RA, Sanchez TG, Kii MA, et al. Repetitive transcranial magnetic stimulation improve tinnitus in normal hearing patients: a double-blind controlled, clinical and neuroimaging outcome study. Eur J Neurol 2010;17(01):38-44

26 Kleinjung T, Steffens T, Sand P, et al. Which tinnitus patients benefit from transcranial magnetic stimulation? Otolaryngol Head Neck Surg 2007;137(04):589-595
27 Langguth B, Kleinjung T, Frank E, et al. High-frequency priming stimulation does not enhance the effect of low-frequency rTMS in the treatment of tinnitus. Exp Brain Res 2008;184(04): 587-591

28 Khedr EM, Rothwell JC, Ahmed MA, El-Atar A. Effect of daily repetitive transcranial magnetic stimulation for treatment of tinnitus: comparison of different stimulus frequencies. J Neurol Neurosurg Psychiatry 2008;79(02):212-215

29 Lorenz I, Müller N, Schlee W, Langguth B, Weisz N. Short-term effects of single repetitive TMS sessions on auditory evoked activity in patients with chronic tinnitus. J Neurophysiol 2010; 104(03):1497-1505

30 Plewnia C, Bartels M, Gerloff C. Transient suppression of tinnitus by transcranial magnetic stimulation. Ann Neurol 2003;53(02): 263-266

31 Plewnia C, Reimold M, Najib A, Reischl G, Plontke SK, Gerloff C. Moderate therapeutic efficacy of positron emission tomographynavigated repetitive transcranial magnetic stimulation for chronic tinnitus: a randomised, controlled pilot study. J Neurol Neurosurg Psychiatry 2007;78(02):152-156

32 Rossi S, De Capua A, Ulivelli M, et al. Effects of repetitive transcranial magnetic stimulation on chronic tinnitus: a randomised, crossover, double blind, placebo controlled study. J Neurol Neurosurg Psychiatry 2007;78(08):857-863

33 Lee SL, Abraham M, Cacace AT, Silver SM. Repetitive transcranial magnetic stimulation in veterans with debilitating tinnitus: a pilot study. Otolaryngol Head Neck Surg 2008;138(03):398-399

34 Wang H, Li B, Wang M, et al. Factor analysis of low-frequency repetitive transcranial magnetic stimulation to the temporoparietal junction for tinnitus. Neural Plast 2016;2016:2814056

35 Meng Z, Liu S, Zheng Y, Phillips JS. Repetitive transcranial magnetic stimulation for tinnitus. Cochrane Database Syst Rev 2011; (10):CD007946 